\title{
Band dispersion in the deep 1s core level of graphene
}

\author{
Silvano Lizzit' ${ }^{1}$, Guillermo Zampieri ${ }^{2}$, Luca Petaccia', Rosanna Larciprete ${ }^{3}$, Paolo Lacovig ${ }^{1,4}$, \\ Emile D. L. Rienks ${ }^{5}$, Gustav Bihlmayer ${ }^{6}$, Alessandro Baraldi ${ }^{4,7}$ and Philip Hofmann ${ }^{5 \star}$
}

\begin{abstract}
It is generally assumed that electrons in deep atomic core states are highly localized and do not participate in the bonding of molecules and solids. This implies well-defined core-level binding energies and the absence of any splitting and band-like dispersion, a fact that is exploited in several powerful experimental techniques, such as X-ray photoemission spectroscopy. Violations of this assumption have been found for only a few small molecules in the gas phase such as $\mathrm{C}_{2} \mathrm{H}_{2}$ or $\mathrm{N}_{2}$ with much stronger bonding and shorter bonding distances than present in solids ${ }^{\mathbf{1 , 2}}$. Here we report the observation of a sizeable band-like dispersion of the $\mathrm{C}$ 1s core level in graphene, a single-layer honeycomb net of carbon atoms that is attracting considerable attention at present in the scientific community ${ }^{3}$. The dispersion is observed as an emission-angle-dependent binding-energy modulation and it is shown that under appropriate conditions only the bonding or antibonding states can be observed. A very similar dispersion is also found by $a b$ initio calculations.
\end{abstract}

The binding-energy modulations of the $\mathrm{C} 1 \mathrm{~s}$ core state are illustrated in Fig. 1b-f. Each panel shows a group of spectra taken at a fixed polar emission angle $\theta$ as a function of azimuthal emission angle $\phi$. Clear shifts of the peak position are observed. Figure 1g shows a comparison of the spectrum taken at normal emission and one taken at $\theta=25^{\circ}$ together with the result of a peak fit to these two spectra.

The binding-energy variation obtained from the peak fitting is given in Fig. $1 \mathrm{~h}$ with the markers corresponding to the spectra in Fig. 1b-f. Strong changes are evident with the largest difference of binding energies spanning $\approx 60 \mathrm{meV}$. The variation is consistent with the point symmetry of the graphene lattice. Figure 1i shows the intensity variations of the peaks, shown as the modulation function. Strong intensity modulations are observed, caused by photoelectron diffraction in the final state. The variations also follow the point symmetry of the graphene lattice, but they do not seem to be correlated with the binding energy in Fig. 1h, with the main structures being in phase for some polar emission angles and out of phase for others.

Although the data of Fig. 1 serve to illustrate the nature of the effect and the fitting procedure, they are insufficient to pin down the physical origin of the modulation. Figure 2 therefore shows a much more extensive data set measured over many polar and azimuthal angles and at different photon energies. Again, a good fit to all of the spectra in the data set was obtained for a single C $1 s$ component using always the same line shape. Note that this excludes the existence of unresolved components, because their intensities would modulate differently, changing the shape of the peak. The left panel of the figure shows the resulting intensity modulation function and the right panel gives the binding-energy modulation. The intensity modulation function is compared to the simulation for a flat, free-standing layer of graphene. The agreement between experiment and simulation is excellent.

The binding-energy modulation, on the other hand, is shown as a function of $\mathbf{k}_{\|}$, the wavevector component parallel to the surface, which is the only relevant wavevector for a two-dimensional system such as graphene. As the portion of the reciprocal space covered by the experiment increases at higher photon energies, a periodic pattern emerges, which, however, does not coincide with the reciprocal lattice mesh. At the origin $\left(\mathbf{k}_{\|}=(0,0)\right)$ the binding energy is always close to its maximum value. The experiments at $h v=400 \mathrm{eV}$ and $500 \mathrm{eV}$ show that the binding energy takes its maximum value also at the next-nearest-neighbour reciprocal lattice points, and the experiments at 600 and $700 \mathrm{eV}$ show that this occurs again at the next-nearest neighbours of these points. At all of the other reciprocal lattice points the binding energy takes its minimum value. The periodic pattern, therefore, is described with two complementary sublattices: the binding energy is at its maximum value at all of the points connected by vectors that are $\sqrt{3}$ times longer than the primitive vectors and rotated by $30^{\circ}$, and it is at its minimum value at all of the other reciprocal lattice points.

There are, in principle, several different mechanisms that could lead to the observed binding-energy variations. The first is the existence of several unresolved components from carbon atoms in a chemically different environment. The relative intensity between these could change because of a final state effect and thereby mimic a peak shift. This seems highly unlikely in the present case, not only because of the excellent agreement with the intensity calculation for a single component, but also because a peak shift of the observed magnitude would essentially require a complete suppression of peak intensities in certain directions, and this is unrealistic for a usual photoelectron diffraction-type modulation. It would also be very unlikely that the mechanism leads to a binding-energy modulation that is periodic in reciprocal space, as seen in Fig. 2.

The second possible explanation is a recoil effect in which some of the photoelectron's energy is used to excite lattice vibrations. This effect has been reported for photoemission from the graphite $\mathrm{C} 1 \mathrm{~s}$ state $^{4}$. For increasing photon energies it leads to a decreasing apparent binding energy because some of the photoelectron's energy remains with the emitting atom. The effect can be of

\footnotetext{
${ }^{1}$ Sincrotrone Trieste, S.S. $14 \mathrm{Km}$ 163.5, 34149 Trieste, Italy, ${ }^{2}$ Centro Atómico Bariloche and Instituto Balseiro, Comisión Nacional de Energía Atómica, 8400-Bariloche, Argentina, ${ }^{3}$ CNR-Institute for Complex Systems, Via Fosso del Cavaliere 100, 00133 Roma, Italy, ${ }^{4}$ Physics Department and CENMAT, University of Trieste, Via Valerio 2, 34127 Trieste, Italy, ${ }^{5}$ Institute for Storage Ring Facilities and Interdisciplinary Nanoscience Center (iNANO), University of Aarhus, 8000 Aarhus C, Denmark, ${ }^{6}$ Institut für Festkörperforschung and Institute for Advanced Simulation, Forschungszentrum Jülich, D-52425 Jülich, Germany, ${ }^{7}$ Laboratorio TASC INFM-CNR, AREA Science Park, S.S. 14 Km 163.5, 34149 Trieste, Italy. *e-mail: philip@phys.au.dk.
} 
a

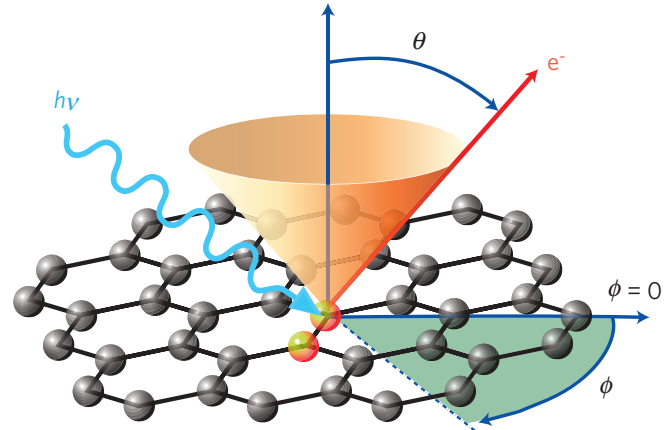

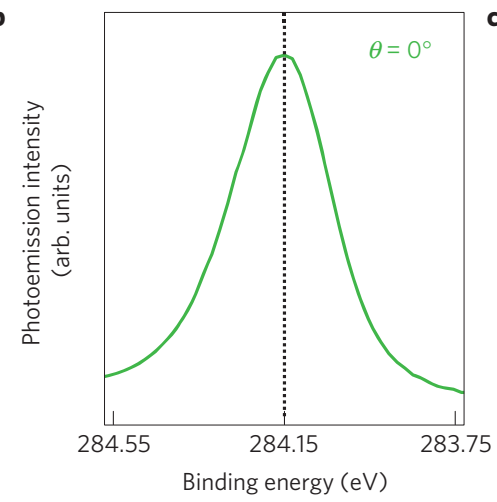

e

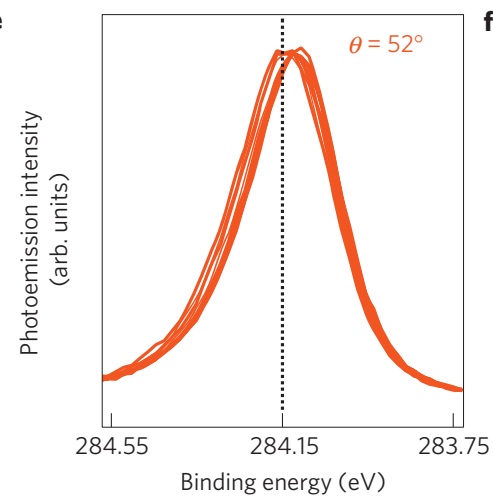

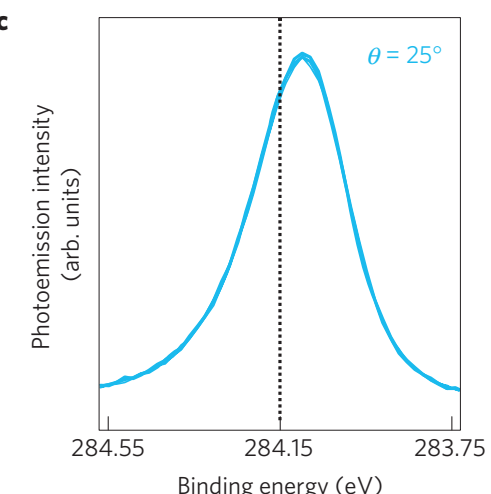

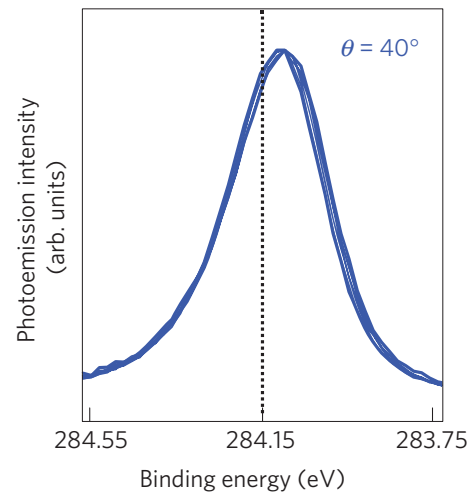

g

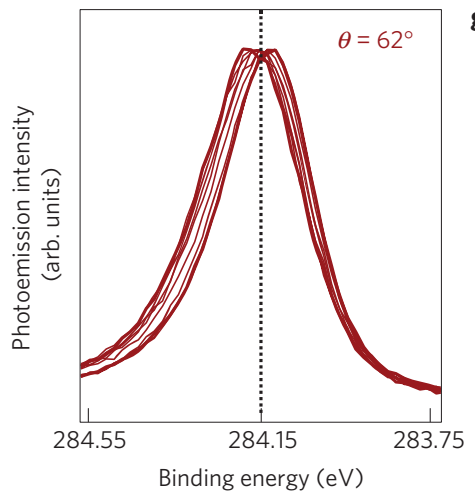

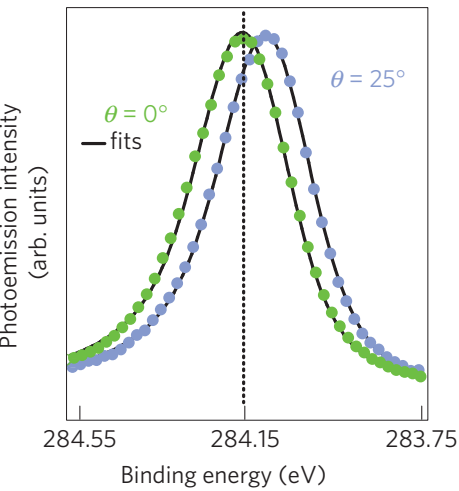

h

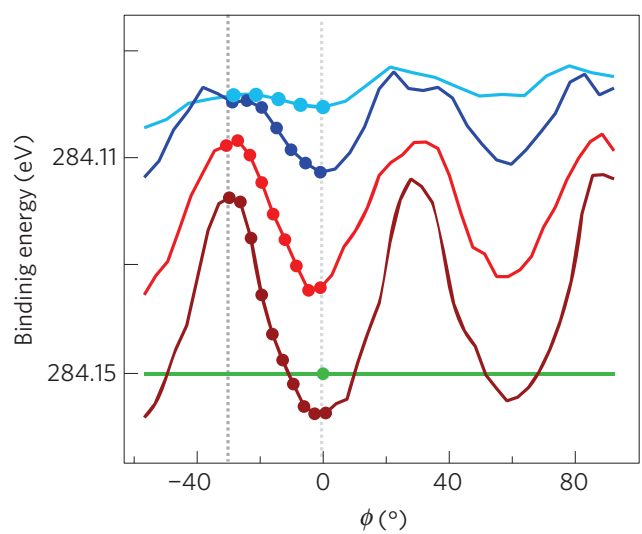

i

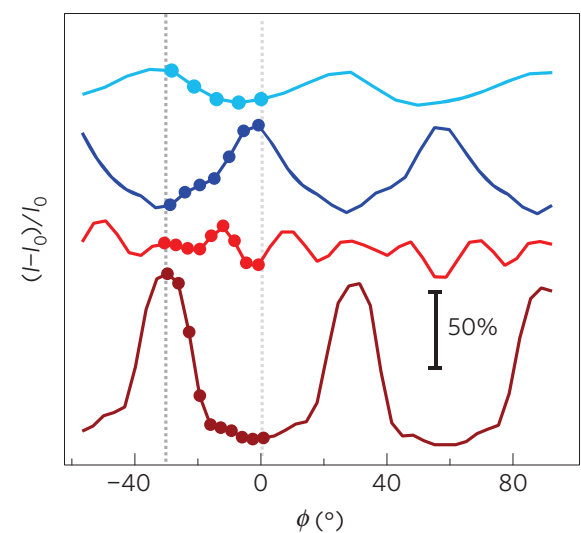

Figure 1 | Manifestation of carbon $1 s$ binding-energy variations in individual X-ray photoemission spectra. a, Schematic of the graphene lattice and the experimental geometry. b-f, $C$ s photoemission spectra taken at a photon energy of $400 \mathrm{eV}$, for fixed polar emission angles $\theta$ in each panel but at different azimuthal emission angles $\phi$. The spectra are all normalized to the same height and shown as a group plot, such that binding-energy variations become evident. The first and last spectrum in a range of azimuthal angles $\phi$ is indicated by a thicker line. $\mathbf{g}$, Comparison of the spectrum taken at $\theta=0^{\circ}$ and one taken at $25^{\circ}$. The lines are the fits through the data points using the line-shape parameters described in the Supplementary Information. $\mathbf{h}, \mathrm{C} 1 \mathrm{~s}$ binding energy required to obtain a good fit for the curves shown above (markers) as well as for the entire azimuthal range measured (lines). The green horizontal line marks the binding energy at normal emission. The binding-energy uncertainty is smaller than $10 \mathrm{meV}$. $\mathbf{i}$, Intensity variation of the $\mathrm{C} 1 \mathrm{~s}$ peak as a function of azimuthal angle. The curves are shifted vertically for clarity. 

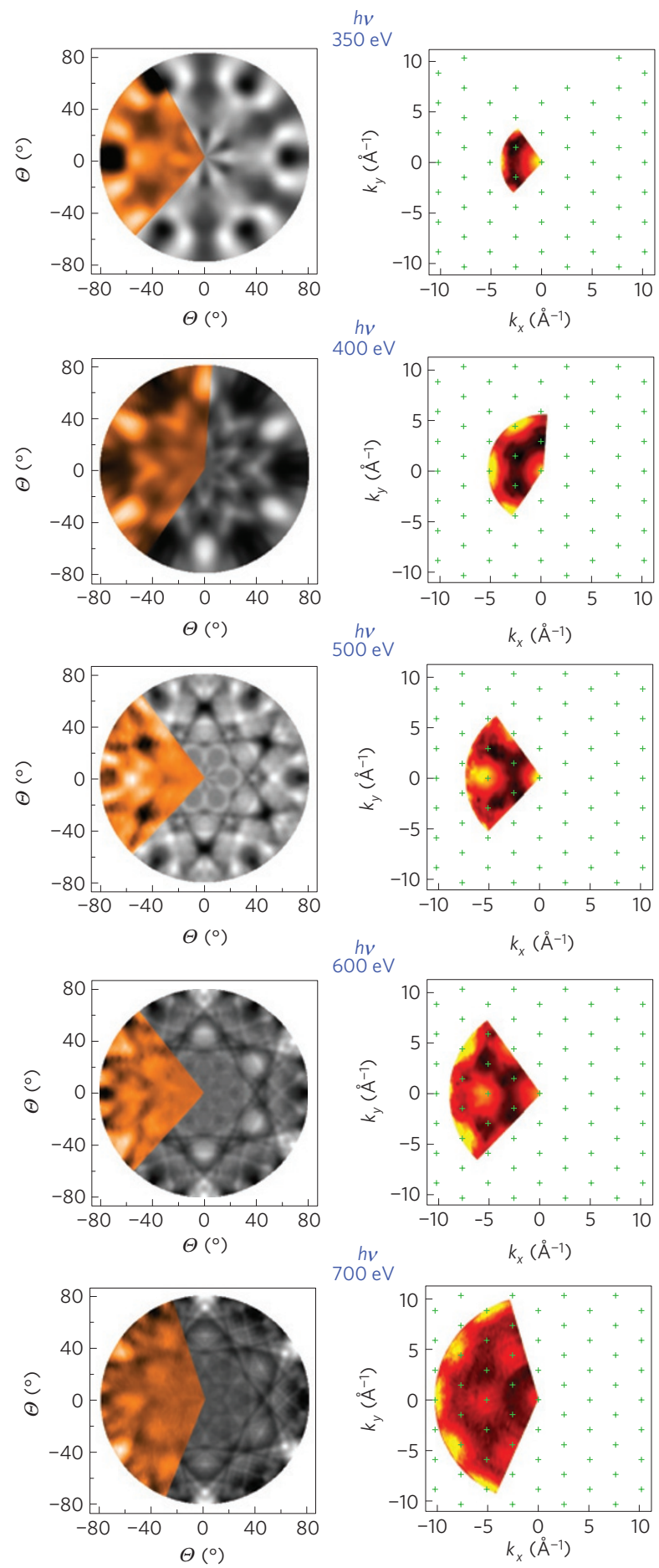

$700 \mathrm{eV}$

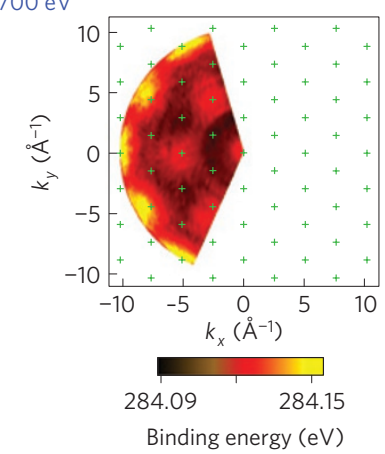

Figure 2 | Carbon 1s intensity and binding-energy variations for large

data sets. Left panel: stereographic projection of the integrated photoemission intensity modulation $\left(I-I_{0}\right) / I_{0}$ as a function of emission angle for scans taken at different photon energies $h v$. The coloured fraction of the disc is the data; the greyscale part is a calculation of the expected intensity. Right panel: the corresponding binding-energy variations obtained from the peak fitting. Note that these variations are shown as a function of the wavevector parallel to the surface rather than emission angle. The binding-energy uncertainty is of the order of $10 \mathrm{meV}$. The green crosses correspond to the reciprocal lattice points of graphene, that is, to the $\bar{\Gamma}$ points. considerable size, several hundred millielectronvolts for photon energies of several kiloelectronvolts, but its magnitude should be insignificant for the energies used here. One would not expect it to lead to any periodic modulation either.

This leaves a third possibility, which is an initial-state effect, that is, a band-like dispersion of the initial state. The simplest conceivable picture for this is the formation of a $\sigma$-type band between the $1 s$ states of the two atoms in the unit cell of graphene, highlighted in orange in Fig. 1a. A tight-binding calculation of such a band is shown in Fig. 3a. The absolute binding energy and the band width are arbitrarily chosen to mimic those observed here. The dispersion shows two bands with the highest energy separation at $\bar{\Gamma}$ and degeneracy at the $\overline{\mathrm{K}}$ point of the two-dimensional Brillouin zone. In the following we refer to these bands, loosely, as the bonding and the antibonding band.

At first sight, it seems hard to reconcile such a dispersion with the experimental observations. The dispersion of Fig. 3a would imply the presence of two components in the $\mathrm{C} 1 s$ peak at $\bar{\Gamma}$ but only one at $\overline{\mathrm{K}}$. As pointed out above, the almost identical peak shape for all emission directions seems to rule out this scenario, as one would expect to observe a single, narrow $\mathrm{C} 1 s$ peak at $\overline{\mathrm{K}}$ and a broad or even split peak at $\bar{\Gamma}$. Furthermore, the $\sigma$-band should be periodic in reciprocal space, for example, the peak position and width should be the same at all $\bar{\Gamma}$ points. According to the right panel of Fig. 2, this is not the case either. Most of the $\bar{\Gamma}$ points marked in the figure appear close to either a maximum or a minimum in the binding energy but clearly the observed periodicity is not the same as that of the reciprocal lattice.

The hypothesis of band dispersion and the experimental data can, however, be reconciled when taking into account a curious interference effect that is caused by the presence of two atoms in the unit cell of graphene. The effect has been studied in detail for the valence band of graphite and graphene $e^{5,6}$.

As an illustration of the interference effect, we have calculated the expected photoemission intensities for all of the antibonding and all of the bonding states. The results are given in Fig. 3b and c, respectively. The strength of the interference effect is evident: in the first Brillouin zone, for instance, emission from the antibonding states is entirely suppressed whereas it is intense from the bonding states. In the neighbouring zones it is the other way round. Figure $3 \mathrm{e}-\mathrm{g}$ shows the emission intensity for smaller energy windows at the bottom of the bonding band, at the top of the antibonding band and just below the Dirac point at $\mathrm{K}$, respectively. In the last case, a constant energy contour shows a triangular shape, as expected for the nonlinear dispersion away from $\overline{\mathrm{K}}$. The photoemission intensity around this triangular contour shows strong variations, which are caused by the interference effect and very similar to the results obtained for the valence $\pi$-band of graphite and graphene $e^{5,6}$.

As already expected from Fig. $3 b, c$, the interference effect is even stronger for emission from the bonding and antibonding states at $\bar{\Gamma}$ (see Fig. 3e,f). The intensity variations of both bands are opposite to each other: for some $\bar{\Gamma}$ points only the bonding band is observed, for others only the antibonding band. For normal emission this is easy to understand: for the bonding band the wavefunctions centred on the two atoms in the unit cell emit in phase and this band is observed. For the antibonding wavefunction, the two atomic wavefunctions emit out of phase, thus suppressing the photoemission.

The presence of this interference effect easily reconciles the hypothesis of a $\sigma$-band formation with the data. First, it explains the fact that the peak shape is very similar for all emission directions. For emission near $\overline{\mathrm{K}}$ the peak is narrow because of the degeneracy of the bands at $\overline{\mathrm{K}}$. At the $\bar{\Gamma}$ points the peak is also narrow, in contrast to naive expectation, because it does not show both the bonding and the antibonding bands but rather only one of them 
a

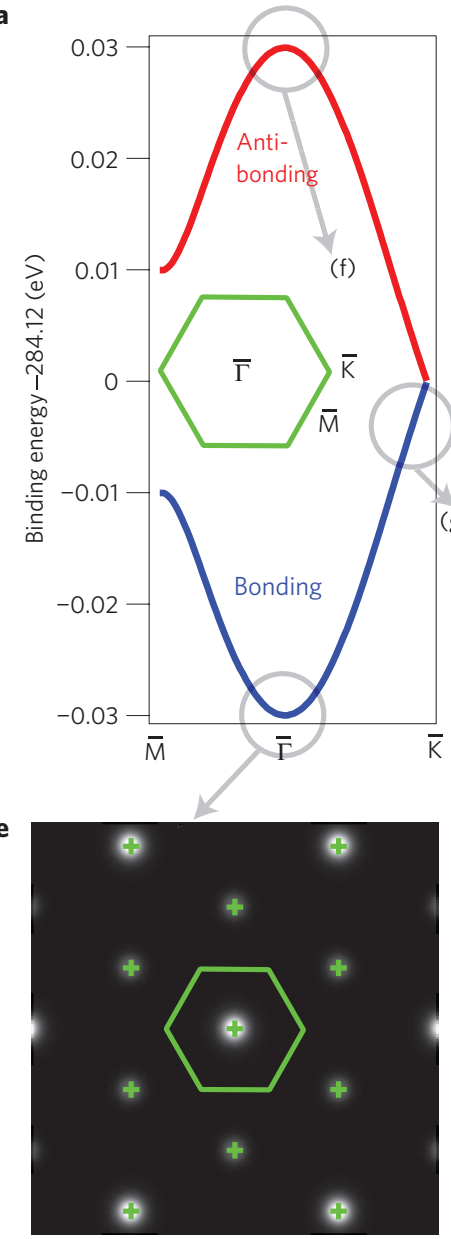

b

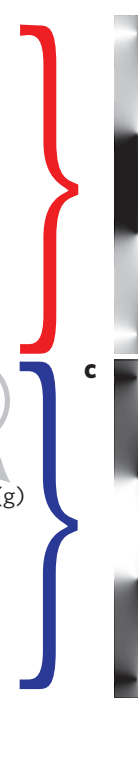

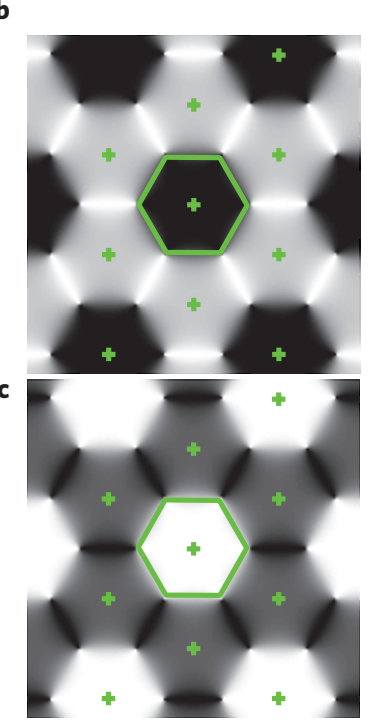

f

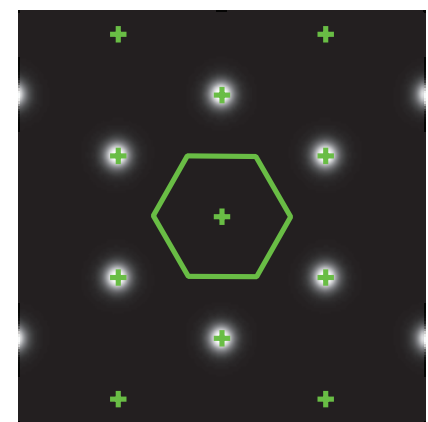

d
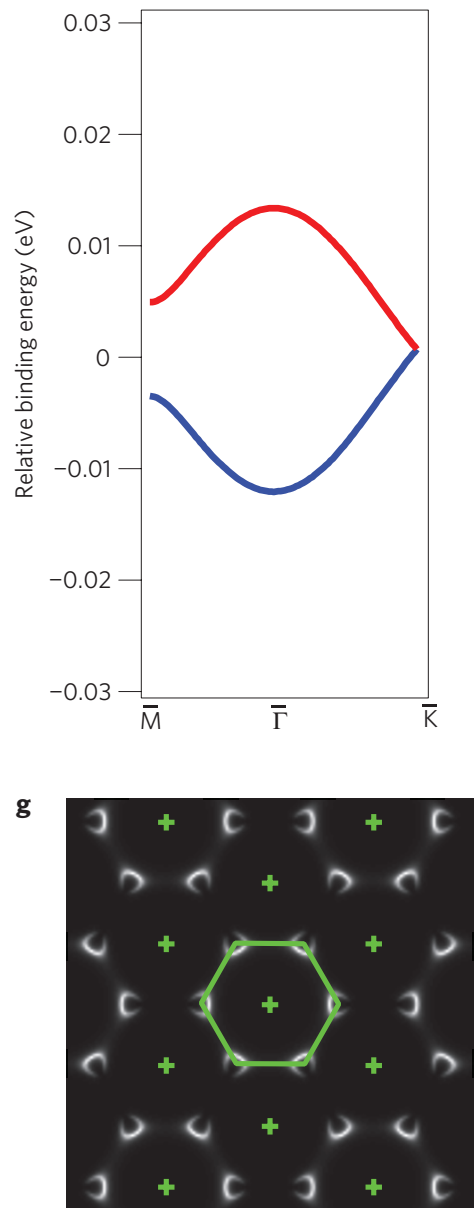

Figure 3 | Calculated C 1s binding-energy variation and interference-induced modulations of the photoemission intensity. a, Tight-binding calculation

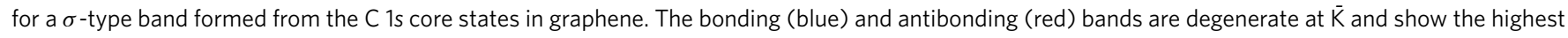
splitting at $\bar{\Gamma}$. The choice of tight-binding parameters was guided by the experimentally observed binding-energy modulations. The inset shows the Brillouin zone of graphene. $\mathbf{b}, \mathbf{c}$, Calculated photoemission intensity from all of the antibonding (b) and bonding (c) states. The greyscale is chosen such that bright corresponds to high intensity. The green crosses mark the reciprocal lattice of graphene and the green hexagon the first Brillouin zone.

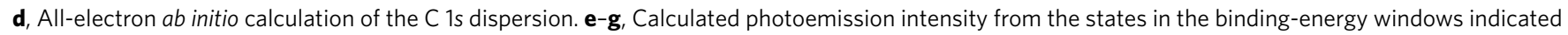

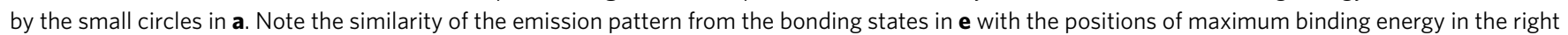
panel of Fig. 2.

at every given $\bar{\Gamma}$ point. The interference effect is also responsible for the seemingly incorrect periodicity of the band dispersion in reciprocal space. The situation is not such that all $\bar{\Gamma}$ points are equivalent, because one observes emission from either the bonding or from the antibonding band. Note that the sign of the observed modulation is consistent with this interpretation, too. The binding energy of the peak observed at normal emission is close to the global maximum of the entire data set, consistent with emission from the bonding $\sigma$-band. In fact, the calculated emission from the bonding band shown in Fig. 3e can be compared directly to the plot of the apparent binding energy in Fig. 2, which is also scaled such that high binding energies are bright.

The fact that only the bonding or the antibonding state is ever observable for a given emission direction permits a simple estimate of the maximum bonding/antibonding energy difference in graphene: the size of this corresponds directly to the difference of observed binding energies at inequivalent $\bar{\Gamma}$ points. Combining all of the available data from $\bar{\Gamma}$ points showing either the bonding or the antibonding band, we evaluate the size of the splitting to be $60 \pm 10 \mathrm{meV}$. Note that this estimate works even if the bonding/antibonding splitting is smaller than the intrinsic width of the individual components. In the case of an incomplete extinction of one of the peak components, we would underestimate the true degree of dispersion. Such an incomplete extinction should, however, also be observable as a change of the peak shape.

The formation of dispersive $\mathrm{C} 1 s$ bands is confirmed by an all-electron $a b$ initio calculation of the electronic structure that we have carried out for a free-standing layer of graphene. The result of this calculation is shown in Fig. $3 \mathrm{~d}$. The dispersion is very similar with a maximum splitting of $25 \mathrm{meV}$, roughly half of the experimental result. Still, the agreement can be judged as fair, considering the small absolute difference in the amount of splitting.

We can also compare the degree of dispersion to the size of the bonding/antibonding splitting in small carbon-containing molecules such as $\mathrm{C}_{2} \mathrm{H}_{2}(\mathrm{C}-\mathrm{C}$ distance of $1.2 \AA$, splitting of $105 \mathrm{meV}$; ref. 1) and $\mathrm{C}_{2} \mathrm{H}_{4}$ (C-C distance of $1.34 \AA$ expected splitting of 20-30 meV; ref. 7) if we assume that the matrix element for hopping between the core electrons on the two carbon atoms depends exponentially on the $\mathrm{C}-\mathrm{C}$ distance and that the size of the splitting scales linearly with the number of nearest neighbours. Assuming a splitting of $105 \mathrm{meV}$ and $25 \mathrm{meV}$ for $\mathrm{C}_{2} \mathrm{H}_{2}$ and $\mathrm{C}_{2} \mathrm{H}_{4}$, respectively, we would expect a total bandwidth of $\approx 30 \mathrm{meV}$ for graphene, very similar to the $a b$ initio result but smaller than that found experimentally. 
Apart from the fundamental importance of our results for the localization of deep core levels in solids, a dependence of the core-level binding energy on the emission angle can have implications for the interpretation of high-resolution corelevel data from graphene, graphite and related materials. The absolute magnitude of the binding-energy variation observed here is appreciable compared with usual chemical shifts and could easily be interpreted incorrectly. Ignoring dispersion effects might have some role in the recent dispute about the existence of a surface core-level shift in graphite ${ }^{8-11}$, but note that the interference effect reported here is inconsistent with the observation of multiple C $1 s$ components.

\section{Received 21 September 2009; accepted 3 February 2010;} published online 14 March 2010

\section{References}

1. Kempgens, B. et al. Core level energy splitting in the $\mathrm{C} 1 \mathrm{~s}$ photoelectron spectrum of $\mathrm{C}_{2} \mathrm{H}_{2}$. Phys. Rev. Lett. 79, 3617-3620 (1997)

2. Hergenhahn, U., Kugeler, O., Rudel, A., Rennie, E. E. \& Bradshaw, A. M. Symmetry-selective observation of the $\mathrm{N}$ 1s shape resonance in $\mathrm{N}_{2}$. J. Phys. Chem. A 105, 5704-5708 (2001).

3. Geim, A. K. Graphene: Status and prospects. Science 324, 1530-1534 (2009).

4. Takata, Y. et al. Recoil effects of photoelectrons in a solid. Phys. Rev. B 75, 233404 (2007).

5. Shirley, E. L., Terminello, L. J., Santoni, A. \& Himpsel, F. J. Brillouin-zone-selection effects in graphite photoelectron angular distributions. Phys. Rev. B 51, 13614-13622 (1995).

6. Mucha-Kruczynski, M. et al. Characterization of graphene through anisotropy of constant-energy maps in angle-resolved photoemission. Phys. Rev. B 77, 195403 (2008).

7. Köppel, H., Gadea, F. X., Klatt, G., Schirmer, J. \& Cederbaum, L. S. Multistate vibronic coupling effects in the K-shell excitation spectrum of ethylene: Symmetry breaking and core-hole localization. J. Chem. Phys. 106, 4415-4429 (1997)

8. Balasubramanian, T., Andersen, J. N. \& Walldén, L. Surface-bulk core-level splitting in graphite. Phys. Rev. B 64, 205420 (2001).

9. Smith, R. A. P., Armstrong, C. W., Smith, G. C. \& Weightman, P. Observation of a surface chemical shift in carbon $1 s$ core-level photoemission from highly oriented pyrolytic graphite. Phys. Rev. B 66, 245409 (2002).

10. Lizzit, S. et al. C 1s photoemission spectrum in graphite(0001). Phys. Rev. B 76, 153408 (2007).

11. Hunt, M. R. C. Surface and bulk components in angle-resolved core-level photoemission spectroscopy of graphite. Phys. Rev. B 78, 153408 (2008).

\section{Acknowledgements}

We gratefully acknowledge stimulating discussions with D. L. Mills, J. G. de Abajo and K. C. Prince. The research leading to these results has received financial support from the Danish National Research Council, the Lundbeck Foundation and the European Community's Seventh Framework Programme (FP7/2007-2013) under grant agreement no. 226716. G.Z. thanks ANPCyT of Argentina and the ICTP Trieste for financial support under the TRIL Programme.

\section{Author contributions}

S.L., G.Z., L.P., R.L., P.L., E.D.L.R., A.B. and P.H. conceived and carried out the measurements. S.L. and P.L. developed the set-up for the measurements. S.L., R.L., P.L. and A.B. implemented the procedure for graphene growth. S.L., L.P., G.Z. and R.L. analysed the X-ray photoemission spectroscopy data. S.L. and G.Z. carried out the photoelectron diffraction model calculations. P.H. and G.Z. carried out the tight-binding calculations. G.B. carried out the ab initio calculations. P.H. wrote the manuscript. All authors extensively discussed the results and commented on the manuscript.

\section{Additional information}

The authors declare no competing financial interests. Supplementary information accompanies this paper on www.nature.com/naturephysics. Reprints and permissions information is available online at http://npg.nature.com/reprintsandpermissions. Correspondence and requests for materials should be addressed to P.H. 